\title{
Classical Scattering with Long Range Forces *
}

\author{
Ira W. Herbst ${ }^{\star \star}$ \\ Department of Physics, The University of Michigan, Ann Arbor, Michigan, USA
}

Received July 30, 1973

\begin{abstract}
We discuss the classical two-body scattering problem for potentials which decrease at infinity like $r^{-\alpha}, 1 \geqq \alpha>0$. We prove existence and uniqueness theorems for scattering orbits parametrized by their asymptotic data. Wave operators are constructed and their properties discussed. We also discuss and prove cluster properties of the $S$-operator.
\end{abstract}

\section{Introduction}

A few years ago, Buslaev and Matveev [1], following Dollard's [2] example in the case of the Coulomb potential, constructed generalized wave operators for the quantum two-body potential scattering problem with potentials which decrease slowly at infinity (i.e. faster than $r^{-\alpha}$ for some $\alpha>0$ ). (See also Ref. [3] for $\alpha \geqq 3 / 4$.) This problem has also been attacked by Alsholm and Kato [4] who rederive the results in [1] with less restrictive assumptions.

In this paper we examine the same problem in classical mechanics. We feel that a more fundamental appreciation of many aspects of the quantum mechanical situation can be gained through an understanding of the corresponding classical problem.

Our formalism is essentially that of Simon [5] who considers the corresponding short range case. (See also Refs. $[6,7]$ where another point of view is taken.) Thus we consider the time development of the system directly in phase space and define a scattering state as one in which the orbit in phase space is unbounded both as $t \rightarrow+\infty$ and $t \rightarrow-\infty$. In Section II we prove existence and uniqueness of scattering solutions parametrized by certain asymptotic data. These are the asymptotic momentum and a three-vector describing how for apart the orbits are asymptotically.

In Section III we introduce additional assumptions concerning the behavior of the derivatives of the potential, $V(x)$, at infinity which enable us to construct a certain quasi-free time evolution $U_{t}^{(0)}$. The operator $U_{i}^{(0)}$ approximates the full dynamics, $U_{t}$, well enough asymptotically so that the transformations $\Omega_{t}=U_{-t} U_{t}^{(0)}$ converge on phase space as

* Research supported in part by the National Science Foundation.

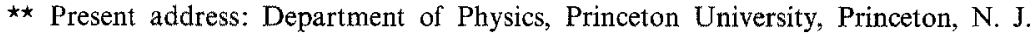
08540 , USA. 
$t \rightarrow \pm \infty$. This defines the wave operators $\Omega_{ \pm}=\lim _{t \rightarrow \pm \infty} \Omega_{t}$. In contrast to the short range case, $U_{t}^{(0)}$ is not an approximation to $U_{t}$ which gives $U_{t} \Omega_{ \pm}-U_{t}^{(0)} \rightarrow 0$ for $t \rightarrow \pm \infty$. We make a conjecture about how this property will be mirrored in quantum mechanics.

With the $S$-operator defined as $S=\Omega_{+}^{-1} \Omega_{-}$, in Section IV we consider the large $|\boldsymbol{a}|$ behavior of $T(-\boldsymbol{a}) S T(\boldsymbol{a})$, where $T(\boldsymbol{a})$ translates the spatial coordinate of a point $Q=(x, p)$ in phase space by an amount $a: T(\boldsymbol{a}) Q=(\boldsymbol{x}+\boldsymbol{a}, \boldsymbol{p})$. In the short range case, the expected result is that for directions, $\hat{a}$, away from the forward direction, $\hat{p}, T(-a) S T(a) Q \rightarrow Q$. These expectations are borne out as we show. In contrast, for the long range case this result is characteristically false. We derive an explicit formula for the limiting behavior for a large class of long range potentials and discuss the probable consequences in the quantum mechanical case.

\section{Existence and Uniqueness of Scattering Solutions}

In the following we are interested in solutions of Newton's equation

$$
\ddot{\boldsymbol{x}}(t)=\boldsymbol{F}(\boldsymbol{x}(t))
$$

with forces satisfying the following conditions which we always will assume hold:

$$
\begin{aligned}
\boldsymbol{F}(\boldsymbol{x}) & =-(\boldsymbol{\nabla} V)(\boldsymbol{x}) \quad \text { with } \quad \lim _{x \rightarrow \infty} V(\boldsymbol{x})=0, \\
\boldsymbol{F}(\boldsymbol{x}) & \leqq k(1+|\boldsymbol{x}|)^{-1-\alpha}, \quad \alpha>0, \quad \alpha^{-1} \neq \text { integer }, \\
\left|\frac{\partial \boldsymbol{F}_{i}(\boldsymbol{x})}{\partial x_{j}}\right| & \leqq k(1+|\boldsymbol{x}|)^{-2-\alpha} .
\end{aligned}
$$

If in Eq. (II.3), $\alpha>1$, results which are analogous to those for short range forces in quantum mechanics (Q.M.) follow if $(1+x)^{-2-\alpha}$ in Eq. (II.4) is replaced by $(1+x)^{-2-\varepsilon}$ with $\varepsilon>0$ [5]. This is essentially the definition of short range force used in Ref. [5]. In the case $\alpha<1$ (which we consider in this paper), the fact that $\alpha$ in Eq. (II.3) is the same as in Eq. (II.4) entails no loss of generality but keeps the bookkeeping to a minimum (note that (II.4) along with $\lim _{x \rightarrow \infty} \boldsymbol{F}(\boldsymbol{x})=0$ implies (II.3)). This is also the reason for demanding $\alpha^{-1} \neq$ integer, for here we avoid the appearance of logarithms in estimates which follow. The prototype force satisfying these conditions comes from the potential $V(x)=\left(1+x^{2}\right)^{-\alpha / 2}$.

Note that condition (II.4) certainly guarantees global existence and uniqueness for the initial value problem [8].

We now define the subsets $\Sigma_{ \pm} \subseteq R^{6}$ corresponding to initial data which lead to scattering solutions of (II.1). 
Thus let $U_{t}$ be the time evolution operator for the system, i.e.

$$
U_{t}\left(x_{0}, p_{0}\right)=(x(t), p(t))
$$

where $\left.(x(0), p(0))=\left(x_{0}, p_{0}\right), p(t)=\dot{x}(t)\right)$, and $x(t)$ satisfies Eq. (II.1). Then we define

$$
\begin{aligned}
\Sigma_{ \pm}= & \left\{\left(\boldsymbol{x}_{0}, \boldsymbol{p}_{0}\right) \in \boldsymbol{R}^{6}: V\left(\boldsymbol{x}_{0}\right)+\frac{1}{2} \boldsymbol{p}_{0}^{2}>0 ;(\boldsymbol{x}(t), \boldsymbol{p}(t))\right. \\
& \text { satisfies } \left.\varlimsup_{t \rightarrow \pm \infty}|\boldsymbol{x}(t)|=\infty\right\} \\
\Sigma_{s}= & \Sigma_{+} \cap \Sigma_{-} .
\end{aligned}
$$

Thus $\Sigma_{ \pm}$contains initial data leading to orbits which are unbounded as $t \rightarrow \pm \infty$, while the scattering states, $\Sigma_{s}$, correspond to orbits unbounded in both directions. (Note that conservation of energy implies that $p(t)$ is always bounded.) The lemma which follows partially characterizes the asymptotic behavior of scattering solutions.

Lemma II.1. Assume (II.2) through (II.4). Then $\left(\boldsymbol{x}_{0}, \boldsymbol{p}_{0}\right) \in \Sigma_{ \pm}$if and only if $(\boldsymbol{x}(t), \boldsymbol{p}(t))=U_{t}\left(\boldsymbol{x}_{0}, \boldsymbol{p}_{0}\right)$ satisfies:

$$
\lim _{t \rightarrow \pm \infty} p(t) \text { exists and is non-zero. }
$$

Proof. Certainly if $\boldsymbol{p}(t) \underset{t \rightarrow-\infty}{\longrightarrow} \neq 0$, then $\left(\boldsymbol{x}_{0}, \boldsymbol{p}_{0}\right) \in \Sigma_{-}$. Conversely if $\left(x_{0}, p_{0}\right) \in \Sigma_{-}$, it is easy to show that for some $t_{0}<0$ and all $t \leqq t_{0}$

$$
|x(t)| \geqq c|t| .
$$

Since the proof of Eq. (II.8) in [5] needs only a slight modification to be applicable here, we do not reproduce it. But given Eq. (II.8) we note that the limit as $t \rightarrow-\infty$ of

$$
p(t)=p_{0}+\int_{0}^{t} d s \boldsymbol{F}(x(s))
$$

exists and defines the asymptotic momentum, $\boldsymbol{p}_{i n}$,

In fact we have

$$
\boldsymbol{p}_{i n}=\boldsymbol{p}_{0}+\int_{0}^{-\infty} d s \boldsymbol{F}(\boldsymbol{x}(\mathrm{s}))
$$

$$
p(t)-p_{i n}=\int_{-\infty}^{t} d s F(x(s))=\mathcal{O}\left(|t|^{-\alpha}\right) .
$$

This proves the $t \rightarrow-\infty$ part of the lemma. The proof for $t \rightarrow+\infty$ is exactly the same.

The parameter, $\boldsymbol{p}_{i n}$, defines half of the asymptotic data at $t=-\infty$. A clue as to where to look for the rest comes from the next lemma, where 
we examine the difference, $x_{2}(t)-x_{1}(t)$, between orbits with the same asymptotic momentum.

Lemma II.2. Assume (II.2) through (II.4). Then if $\left(x_{1}(0), p_{1}(0)\right)$ and $\left(\boldsymbol{x}_{2}(0), \boldsymbol{p}_{2}(0)\right)$ are in $\Sigma_{ \pm}$, and both satisfy

then as $t \rightarrow \pm \infty$

$$
\lim _{t \rightarrow \pm \infty} p_{i}(t)-p=0 ; \quad i=1,2
$$

$$
p_{1}(t)-p_{2}(t)=\mathscr{O}\left(|t|^{-1-\alpha}\right)
$$

and there exists a three-vector a such that

$$
\left|x_{1}(t)-x_{2}(t)-a\right|=\mathscr{O}\left(|t|^{-\alpha}\right) .
$$

If $a=0$, then $x_{1}(t) \equiv x_{2}(t)$.

Proof. We prove the lemma for $\left(\boldsymbol{x}_{i}(0), \boldsymbol{p}_{i}(0)\right) \in \Sigma_{-}$. Let $\boldsymbol{\Delta}(t)=\boldsymbol{x}_{2}(t)$ $-x_{1}(t)$. Eqs. (II.10) and (II.11) tell us that $|\dot{d}(t)|=\mathscr{O}\left(|t|^{-\alpha}\right)$ and thus $|A(t)|=\mathcal{O}\left(1+|t|^{1-\alpha}\right)$. Note that

$$
\dot{\boldsymbol{\Delta}}(t)=\int_{-\infty}^{t} d s\left(\boldsymbol{F}\left(\boldsymbol{x}_{2}(s)\right)-\boldsymbol{F}\left(\boldsymbol{x}_{1}(s)\right)\right)
$$

and thus for $t$ sufficiently negative

$$
|\dot{\Delta}(t)| \leqq \beta \int_{-\infty}^{t} d s \frac{|\Delta(s)|}{(1+|s|)^{2+\alpha}} .
$$

Here we have used Eqs. (II.4) and (II.8). We now reason by induction to show $|\boldsymbol{A}(t)|$ is bounded for $t<0$ : Assume $|\dot{\Delta}(t)|=\mathcal{O}\left(|t|^{-n \alpha}\right)$ for $t \rightarrow-\infty$ with $n \alpha<1$. (We already know this for $n=1$.) Then $|\boldsymbol{A}(s)|=\mathcal{O}\left(1+|s|^{1-n \alpha}\right.$ ), and Eq. (II.15) implies $|\dot{\Delta}(t)|=\mathcal{O}\left(|t|^{-(n+1) \alpha}\right)$. This immediately gives the result that $|\Delta(t)|$ is bounded for $t<0$, and reference to Eq. (II.15) then results in Eqs. (II.12) and (II.13). The last statement of the lemma can be proved in the following way: Let $\|\boldsymbol{A}\|_{T}=\sup _{t \leqq T}|\boldsymbol{\Delta}(t)|$. For $T$ sufficiently negative, and for $t \leqq T$ Eq. (II.15) gives

$$
|\dot{\Delta}(t)| \leqq \beta(1+\alpha)^{-1}\|\Delta\|_{T}(1+|t|)^{-1-\alpha}
$$

and since by assumption $\Delta(t) \rightarrow 0$ as $t \rightarrow-\infty$, integration gives

$$
\|\boldsymbol{A}\|_{T} \leqq \beta \alpha^{-1}(1+\alpha)^{-1}\|\boldsymbol{\Delta}\|_{T}(1+\mid T)^{-\alpha} .
$$

Thus if $|T|$ is large enough, $\|\boldsymbol{A}\|_{T} \leqq \varrho\|\boldsymbol{A}\|_{T}$ with $\varrho<1$ and thus $\Delta(t)=0$ if $t \leqq T$. The last statement of the lemma then follows from the uniqueness of the initial value problem.

This lemma has the consequence that if we know one orbit, $\left(x_{0}(t), p_{0}(t)\right)$, with initial values in $\Sigma_{-}$and asymptotic momentum $p_{i n}$, then all other 
orbits with the same asymptotic momentum are uniquely specified by the asymptotic difference, $\boldsymbol{a}=\lim _{t \rightarrow-\infty}\left(\boldsymbol{x}(t)-\boldsymbol{x}_{0}(t)\right)$. However if the force is short range, it is not neccesary to subtract from $x(t)$ another solution to Newton's equation with the same asymptotic momentum. It is well known that an approximate solution, namely a solution to the free equation, will do the job. Thus in this case, the limit

$$
\lim _{t \rightarrow-\infty}\left(x(t)-p_{i n} t\right)
$$

exists and along with $\boldsymbol{p}_{\text {in }}$ uniquely specifies the orbit [5]. Even though the above limit characteristically fails to exist in the long range case, a function $z^{-}(p, t)$, analogous to the function $p t$ can be found for which

$$
\lim _{t \rightarrow-\infty}\left(x(t)-z^{-}\left(p_{i n}, t\right)\right)
$$

does exist and which along with $\boldsymbol{p}_{\text {in }}$ also uniquely specifies the orbit. To find this function, it is enough to iterate Eq. (II.10). Thus let

$$
N=[1 / \alpha]
$$

and define for $n=1,2, \ldots, N$ and $p \neq 0$

$$
\begin{gathered}
\dot{z}_{0}^{ \pm}(p, t)=p ; \quad z_{n}^{ \pm}(p, 0)=0 ; \quad \dot{z}_{n}^{ \pm}(p, t)=p+\int_{ \pm \infty}^{t} d s F\left(z_{n-1}^{ \pm}(p, s)\right), \\
z^{ \pm}(p, t) \equiv z_{N}^{ \pm}(p, t) .
\end{gathered}
$$

We now state the main results of this section.

Theorem II.1. Assume (II.2) through (II.4). Then for each $(\boldsymbol{a}, \boldsymbol{p}) \in \Sigma$ $\equiv\left\{(a, p) \in R^{6}: p \neq 0\right\}$ there exists a unique

$$
\left(\boldsymbol{x}_{0}^{ \pm}, \boldsymbol{p}_{0}^{ \pm}\right) \in \Sigma_{ \pm} \quad \text { with } \quad U_{t}\left(\boldsymbol{x}_{0}^{ \pm}, \boldsymbol{p}_{0}^{ \pm}\right)=\left(\boldsymbol{x}_{(\boldsymbol{a}, \boldsymbol{p})}^{ \pm}(t), \dot{\boldsymbol{x}}_{(\boldsymbol{a}, \boldsymbol{p})}^{ \pm}(t)\right)
$$

satisfying

$$
\begin{array}{r}
\lim _{t \rightarrow \pm \infty}\left|\dot{x}_{(\boldsymbol{a}, \boldsymbol{p})}^{ \pm}(t)-\boldsymbol{p}\right|=0, \\
\lim _{t \rightarrow \pm \infty}\left|\boldsymbol{x}_{(\boldsymbol{a}, \boldsymbol{p})}^{ \pm}(t)-z^{ \pm}(\boldsymbol{p}, t)-\boldsymbol{a}\right|=0 .
\end{array}
$$

Conversely, if $\left(\boldsymbol{x}_{0}^{ \pm}, \boldsymbol{p}_{0}^{ \pm}\right) \in \Sigma_{ \pm}$, then $U_{t}\left(\boldsymbol{x}_{0}^{ \pm}, \boldsymbol{p}_{0}^{ \pm}\right)=\left(\boldsymbol{x}_{(\boldsymbol{a}, \boldsymbol{p})}^{ \pm}(t), \boldsymbol{x}_{(\boldsymbol{a}, \boldsymbol{p})}^{ \pm}(t)\right)$ for some $(a, p) \in \Sigma$.

Proof. We prove only the $t \rightarrow-\infty$ part of the theorem, omitting the superscript - for brevity. We first state without proof a property of the last two members of the sequence $z_{n}$, which we will need to prove the theorem: If $|\boldsymbol{p}| \geqq \varepsilon>0$, then there exist $c, \delta, t_{0}$ all depending on $\varepsilon$, such that 
for $t \leqq t_{0}$

$$
\begin{aligned}
\left|z_{N}(p, t)-z_{N-1}(p, t)\right| & \leqq c|t|^{1-N \alpha}, \\
\left|z_{N}(p, t)\right| & \geqq \delta|t| .
\end{aligned}
$$

These estimates can easily be proved by induction and hold uniformly in $p$ for $|\boldsymbol{p}| \geqq \varepsilon$. We now consider the integral equation

$$
y(t)=(\boldsymbol{w}(\boldsymbol{y}))(t)=\int_{-\infty}^{t} d \tau \int_{-\infty}^{\tau} d s\left[\boldsymbol{F}\left(z_{N}(\boldsymbol{p}, s)+\boldsymbol{a}+\boldsymbol{y}(s)\right)-\boldsymbol{F}\left(z_{N-1}(\boldsymbol{p}, s)\right)\right]
$$

and the set $C_{T}$ of continuous functions $y:(-\infty, T] \rightarrow \boldsymbol{R}^{3}$ satisfying $\|\boldsymbol{y}\|_{T}=\sup _{t \in\{-\infty, T]}|\boldsymbol{y}(t)| \leqq 1$. We will show that for $T$ sufficiently negative, $\psi$ is a contraction mapping of $C_{T}$ into itself. Thus consider the integrand of Eq. (II.25) for $y \in C_{T}$ :

$$
\begin{aligned}
I(\boldsymbol{y}, s)= & \boldsymbol{F}\left(z_{N}(\boldsymbol{p}, s)+\boldsymbol{a}+\boldsymbol{y}\right)-\boldsymbol{F}\left(z_{N+1}(\boldsymbol{p}, s)\right) \\
= & {\left[\boldsymbol{F}\left(z_{N}(\boldsymbol{p}, s)+\boldsymbol{a}+\boldsymbol{y}\right)-\boldsymbol{F}\left(z_{N}(\boldsymbol{p}, s)\right)\right]+\left[\boldsymbol { F } \left(z_{N}(\boldsymbol{p}, s)\right.\right.} \\
& \left.-\boldsymbol{F}\left(\boldsymbol{z}_{N-1}(\boldsymbol{p}, s)\right)\right] .
\end{aligned}
$$

For $s$ sufficiently negative, this can be estimated from Eqs. (II.4), (II.23) and (II.24) with the result

$$
I(\boldsymbol{y}, s) \leqq c_{1}|s|^{-1-(N+1) \alpha} .
$$

Thus for $T$ sufficiently negative,

$$
\|\boldsymbol{\psi}(\boldsymbol{y})\|_{T} \leqq c_{2}|T|^{1-(N+1) x} .
$$

Similarly for $y_{1}, y_{2} \in C_{T}$

$$
\left\|\psi\left(y_{2}\right)-\varphi\left(y_{1}\right)\right\|_{T} \leqq c_{3}|T|^{-\alpha}\left\|y_{1}-y_{2}\right\|_{T} .
$$

Note that the estimates (II.28) and (II.29) are uniform in (a,p) for $|\boldsymbol{p}| \geqq \varepsilon$, $|a| \leqq a_{0}$. If $|T|$ is chosen large enough, then $\psi: C_{T} \rightarrow C_{T}$ and is a contraction. Thus by the contraction mapping theorem $\psi$ has a unique fixed point $y \in C_{T}$. Defining

we note that

$$
x_{(a, p)}(t)=z_{N}(p, t)+a+y(t),
$$

$$
\dot{\boldsymbol{x}}_{(\boldsymbol{a}, \boldsymbol{p})}(t)=\boldsymbol{p}+\int_{-\infty}^{t} d s \boldsymbol{F}\left(\boldsymbol{x}_{(\boldsymbol{a}, \boldsymbol{p})}(s)\right),
$$

and thus Eqs. (II.21) and (II.22) easily follow. Uniqueness follows from Lemma II. 2 since if $\dot{\boldsymbol{x}}(t)-\boldsymbol{p} \rightarrow 0$ and $\boldsymbol{x}(t)-\boldsymbol{z}_{N}(\boldsymbol{p}, t)-\boldsymbol{a} \rightarrow 0$, then $x(t)-x_{(a, p)}(t) \rightarrow 0$. Finally if $\left(x_{0}, p_{0}\right) \in \Sigma_{-}$, then $U_{t}\left(x_{0}, p_{0}\right)=(x(t), p(t))$ satisfies $p(t) \rightarrow p$ for some $\boldsymbol{p} \neq 0$, and in addition, according to Lemma II.2, 
for any $\boldsymbol{a}$ there exists $\boldsymbol{b}$ such that $\boldsymbol{x}_{(\boldsymbol{a}, \boldsymbol{p})}(t)-\boldsymbol{x}(t)+\boldsymbol{b} \rightarrow 0$. Thus if $\boldsymbol{c}=\boldsymbol{a}+\boldsymbol{b}$, we easily see that

$$
\left|x(t)-x_{(c, p)}(t)\right| \leqq\left|x_{(e, p)}(t)-x_{(a, p)}(t)-b\right|+\left|x_{(a, p)}(t)-x(t)+b\right| \rightarrow 0
$$

and thus again by Lemma II.2, $\boldsymbol{x}(t) \equiv \boldsymbol{x}_{(\boldsymbol{c}, \boldsymbol{p})}(t)$. Hence the theorem is proved.

In order to prepare for the construction of wave operators in the next section, we want to discuss the content of Theorem II. 1 in more formal terms: We have parametrized the orbits which are unbounded as $t \rightarrow \pm \infty$ using the approximate dynamics $E_{t}^{(0)}$,

$$
E_{t}^{(0)}(\boldsymbol{\alpha}, \boldsymbol{\beta})=\left(\boldsymbol{\alpha}+\boldsymbol{z}^{ \pm}(\boldsymbol{\beta}, t), \boldsymbol{\beta}\right), \quad \pm t>0 .
$$

Note that $E_{t}^{(0)}$ is a measure preserving transformation on phase space (it is a momentum dependent translation of the spatial coordinate) which does not change the momentum. It also commutes with the free dynamics $S_{t}^{(0)}$,

$$
S_{t}^{(0)}(\boldsymbol{\alpha}, \boldsymbol{\beta})=(\boldsymbol{\alpha}+\boldsymbol{\beta} t, \boldsymbol{\beta}) .
$$

For any $(a, p) \in \Sigma$, there is a unique $\left(x_{0}^{ \pm}, p_{0}^{ \pm}\right) \in \Sigma_{ \pm}$(this defines the maps $\left.W_{ \pm}(\boldsymbol{a}, \boldsymbol{p})=\left(\boldsymbol{x}_{0}^{ \pm}, \boldsymbol{p}_{0}^{ \pm}\right)\right)$such that

$$
\lim _{t \rightarrow \pm \infty}\left(U_{t}\left(x_{0}^{ \pm}, p_{0}^{ \pm}\right)-E_{t}^{(0)}(\boldsymbol{a}, \boldsymbol{p})\right)=0
$$

or in other words, on $\Sigma$

$$
\lim _{t \rightarrow \pm \infty}\left(U_{t} W_{ \pm}-E_{t}^{(0)}\right)=0 .
$$

The maps $W_{ \pm}: \Sigma \rightarrow \Sigma_{ \pm}$are $1-1$ and onto; it is also straightforward to show that $W_{ \pm}$intertwines $U_{t}$ and $S_{t}^{(0)}$ :

$$
U_{t} W_{ \pm}=W_{ \pm} S_{t}^{(0)} \text {. }
$$

In addition, with a further restriction on the derivatives of $\boldsymbol{F}(\boldsymbol{x})$ it can be shown that the $W_{ \pm}$preserve Lebesgue measure. (We do not give the proof of this fact.)

We have introduced the transformations $W_{ \pm}$because if the forces are short range, the wave operators also satisfy (II.35) and (II.36) (with $E_{t}^{(0)}$ replaced by $\left.S_{t}^{(0)}\right)$ and also preserve Lebesgue measure. Thus one might be tempted to call the $W_{ \pm}$generalized wave operators. We do not follow this procedure for the following reasons. The operators $E_{t}^{(0)}$, while they are measure preserving, are not in general canonical transformations, i.e. they do not preserve Poisson brackets. This means that there is in general no unitary analog of $E_{t}^{(0)}$ in Q.M. The fact that $E_{t}^{(0)}$ is not in general a canonical transformation has the consequence that the 
$W_{ \pm}$are also not canonical transformations and thus very likely have no isometric analogs in Q.M.

In the next section we will construct wave operators which are canonical transformations, and which do have isometric analogs in Q.M. However, this will be accomplished only at the expense of additional restrictive assumptions on the potential.

\section{The Existence of Wave Operators}

In analogy with Q.M. where for short range interactions the wave operators $\Omega_{ \pm}$are defined as the strong limits of $e^{i H t} e^{-i H_{0} t}$ as $t \rightarrow \pm \infty$, we seek a "quasi-free" dynamics $U_{t}^{(0)}$ which approximates the full dynamics $U_{t}$ well enough so that

$$
\Omega_{t}=U_{t}^{-1} U_{t}^{(0)}
$$

will converge as $t \rightarrow \pm \infty$ :

$$
\Omega_{ \pm}=\lim _{t \rightarrow \pm \infty} \Omega_{t} .
$$

We impose two requirements on $U_{t}^{(0)}$ :

(i) $U_{t}^{(0)}$ does not change the momentum.

(ii) $U_{i}^{(0)}$ is a canonical transformation.

The first requirement is the simplest way of guaranteeing that the parameter $\boldsymbol{p}$ in the expression $\Omega_{-}(\boldsymbol{a}, \boldsymbol{p})=\left(\boldsymbol{x}_{0}, \boldsymbol{p}_{0}\right)$ is the asymptotic momentum (as $t \rightarrow-\infty)$ associated with the orbit $U_{t}\left(x_{0}, p_{0}\right)=(x(t), p(t))$. The second requirement is a natural one which in Q.M. would guarantee the unitarity of $U_{t}^{(0)}$. Note that if $H^{\prime}=H_{0}+U$ is the Hamiltonian associated with $U_{t}^{(0)}$, then because of the first requirement $U$ must be $x$ independent. Thus $U=U(p, t)$. Hamilton's equations then become

Thus

$$
\dot{p}=0, \quad \dot{x}=p+\nabla_{p} U(p, t) .
$$

where

$$
U_{t}^{(0)}(\boldsymbol{a}, \boldsymbol{p})=(\boldsymbol{a}+\boldsymbol{x}(\boldsymbol{p}, t), \boldsymbol{p})
$$

$$
x(p, t)=p t+\int_{0}^{t} d s \nabla_{p} U(p, s)
$$

Perhaps the easiest way of finding an appropriate $U(\boldsymbol{p}, t)$ is to consider the transformation $\Omega_{t}^{-1}$ which when applied to $\left(x_{0}, p_{0}\right) \in \Sigma_{-}$should give a convergent result as $t \rightarrow-\infty$. The sequence of equalities

$$
\begin{aligned}
\Omega_{t}^{-1}\left(x_{0}, p_{0}\right) & =U_{t}^{(0)-1} U_{t}\left(x_{0}, p_{0}\right)=U_{t}^{(0)-1}(x(t), p(t)) \\
& =(x(t)-x(p(t), t), p(t))
\end{aligned}
$$


tells us that we are interested in the convergence of the function

$$
\boldsymbol{r}(t)=\boldsymbol{x}(t)-\boldsymbol{x}(\boldsymbol{p}(t), t)
$$

as $t \rightarrow-\infty$. We have

$$
\dot{r}_{j}(t)=\left[p_{j}-\frac{\partial x_{j}}{\partial p_{i}}(\boldsymbol{p}, t) F_{i}(\boldsymbol{x}(t))-\frac{\partial x_{j}}{\partial t}(\boldsymbol{p}, t)\right]_{\boldsymbol{p}=\boldsymbol{p}(t)}
$$

or using Eq. (III.4)

$$
\dot{r}_{j}(t)=-\left[\frac{\partial x_{j}}{\partial p_{i}}(\boldsymbol{p}, t) F_{i}(\boldsymbol{x}(t))+\frac{\partial U}{\partial p_{j}}(\boldsymbol{p}, t)\right]_{\boldsymbol{p}=\boldsymbol{p}(t)} .
$$

If we had $U(\boldsymbol{p}, t)=V(\boldsymbol{x}(\boldsymbol{p}, t))$ then Eq. (III.6) would become

$$
\dot{r}_{j}(t)=\left\{\frac{\partial x_{j}}{\partial p_{i}}(\boldsymbol{p}, t)\left[F_{i}(\boldsymbol{x}(\boldsymbol{p}, t))-F_{i}(\boldsymbol{x}(t))\right]\right\}_{\boldsymbol{p}=\boldsymbol{p}(t)},
$$

and this has a reasonable chance of decreasing fast enough as $t \rightarrow-\infty$ to make $\lim _{t \rightarrow-\infty} r(t)$ exist. Specifically if for $|t|$ large enough

$$
\left|\frac{\partial x_{j}}{\partial p_{i}}(\boldsymbol{p}, t)\right| \leqq c_{1}|t|, \quad|\boldsymbol{x}(\boldsymbol{p}, t)| \geqq c_{2}|t|,
$$

then Eq. (III.7) implies

$$
|\dot{r}(t)| \leqq c|r(t)||t|^{-1-\alpha}
$$

which in turn means $|\dot{r}(t)|=\mathscr{O}\left(|t|^{-1-\alpha}\right)$, and this is certainly enough to guarantee the existence of $\lim _{t \rightarrow-\infty} r(t)$. Unfortunately Eq. (III.4) together with $U(\boldsymbol{p}, t)=V(\boldsymbol{x}(\boldsymbol{p}, t))$ imply we must solve the equation

$$
\boldsymbol{x}(\boldsymbol{p}, t)=\boldsymbol{p} t+\boldsymbol{\nabla}_{\boldsymbol{p}} \int_{0}^{t} d s V(\boldsymbol{x}(\boldsymbol{p}, s))
$$

or in terms of $U$

$$
U(\boldsymbol{p}, t)=V\left(\boldsymbol{p} t+\int_{0}^{t} d s \boldsymbol{\nabla}_{\boldsymbol{p}} U(\boldsymbol{p}, s)\right) .
$$

This is exactly the equation arrived at in Ref. [1] where the authors treat the Q.M. case. Because we do not know under what circumstances this equation has solutions, we follow the example of Ref. [1] and iterate Eq. (III.9). Thus we define for $p \neq 0$,

$$
\begin{aligned}
& U_{0}(\boldsymbol{p}, t)=0 \\
& U_{k}(\boldsymbol{p}, t)=V\left(\boldsymbol{p} t+\int_{0}^{t} d s \nabla_{\boldsymbol{p}} U_{k-1}(\boldsymbol{p}, s)\right) \quad k=1,2, \ldots, N+1
\end{aligned}
$$


where $N=[1 / \alpha]$. Also define

$$
\boldsymbol{x}_{k}(\boldsymbol{p}, t)=\boldsymbol{p} t+\int_{0}^{t} d s \nabla_{p} U_{k}(\boldsymbol{p}, s) \quad k=0,1, \ldots, N .
$$

We thus have

$$
\boldsymbol{x}_{k}(\boldsymbol{p}, t)=\boldsymbol{p} t+\int_{0}^{t} d s \boldsymbol{\nabla}_{\boldsymbol{p}} V\left(\boldsymbol{x}_{k-1}(\boldsymbol{p}, s)\right), \quad k=1, \ldots, N .
$$

Finally let

$$
\boldsymbol{x}(\boldsymbol{p}, t)=\boldsymbol{x}_{N}(\boldsymbol{p}, t) ; \quad U(\boldsymbol{p}, t)=U_{N}(\boldsymbol{p}, t) .
$$

Since the $n^{\text {th }}$ partial, $D^{n} U_{k}(\boldsymbol{p}, t)$ involves $\left(D^{n+k} V\right)(\boldsymbol{x})$, it is clear that for all quantities to be well defined we need a condition like $V \in C^{N+1}\left(R^{3}\right)$. In fact we find that if the sequence is to have the required properties, much more is required. Thus we assume in all of the following,

$V \in C^{N+2}\left(R^{3}\right) ; \quad\left|\left(D^{n} V\right)(x)\right| \leqq c|x|^{-\alpha-n}, \quad n=0,1, \ldots, N+2$.

We now list the properties of the $U_{n}$ and $x_{n}$ which we will need. These follow from Eq. (III.14) and can be proved by induction. (See also Ref. [1].) For $|\boldsymbol{p}| \geqq \varepsilon>0$

$$
\begin{aligned}
& \left|\left(D^{n} U_{N+1}\right)(\boldsymbol{p}, t)-\left(D^{n} U_{N}\right)(\boldsymbol{p}, t)\right| \leqq c(1+|t|)^{-\alpha(N+1)} ; \quad n=0,1,2, \\
& \left|\left(D^{n} \boldsymbol{x}\right)(\boldsymbol{p}, t)\right| \leqq c(1+|t|) ; \quad n=0,1,2 \quad\left|\boldsymbol{x}_{N}(\boldsymbol{p}, t)-\boldsymbol{p} t\right| \leqq c|t|^{1-\alpha} .
\end{aligned}
$$

The proof of the following theorem shows how the heuristic argument based on Eq. (III.8) needs to be modified.

Theorem III.1. Assume (III.14). Then the transformations $\lim _{t \rightarrow \pm \infty} \Omega_{t}^{-1}$, exist and define $1-1$ maps $\Omega_{ \pm}^{-1}$ of $\Sigma_{ \pm}$onto $\Sigma . \Omega_{ \pm}$intertwines $S_{t}^{(0)}$ and $U_{t}$

$$
\Omega_{ \pm} S_{t}^{(0)}=U_{t} \Omega_{ \pm} \text {. }
$$

In addition if $\left(\boldsymbol{x}_{1}(0), \boldsymbol{p}_{1}(0)\right)$ and $\left(\boldsymbol{x}_{2}(0), \boldsymbol{p}_{2}(0)\right)$ are in $\Sigma_{ \pm}$and both satisfy Eq. (II.11), then

$\Omega_{ \pm}^{-1}\left(x_{1}(0), p_{1}(0)\right)-\Omega_{ \pm}^{-1}\left(x_{2}(0), p_{2}(0)\right)=\lim _{t \rightarrow \pm \infty}\left(x_{1}(t)-x_{2}(t), 0\right)$.

Remark. Eq. (III.18) serves to give some meaning to the parameter $a$ which occurs in the expression $\Omega_{+}^{-1}\left(x_{0}, p_{0}\right)=(a, p)$. Although we will not demonstrate it, if $V(x)$ is a central potential, the component of $a$ perpendicular to $p, a_{\perp}$, is just the impact parameter, $\lim _{t \rightarrow \pm \infty} x_{\perp}(t)$. Of course the parameter $\boldsymbol{p}$ is always the asymptotic momentum. 
Proof. The proof of Theorem III.1 is simple. We consider the behavior of $r(t)$ in the expression

$$
\Omega_{t}^{-1}\left(\boldsymbol{x}_{0}, \boldsymbol{p}_{0}\right)=(r(t), \boldsymbol{p}(t)) .
$$

Where as before $r(t)=\boldsymbol{x}(t)-\boldsymbol{x}(\boldsymbol{p}(t), t)$. Instead of Eq. (III.7) we have

$$
\begin{aligned}
\dot{r}_{j}(t)= & {\left[\frac{\partial x_{j}}{\partial p_{i}}(\boldsymbol{p}, t)\left(F_{i}(\boldsymbol{x}(\boldsymbol{p}, t))-F_{i}(\boldsymbol{x}(t))\right)\right.} \\
& \left.+\frac{\partial}{\partial p_{j}}\left(U_{N+1}(\boldsymbol{p}, t)-U_{N}(\boldsymbol{p}, t)\right)\right]_{\boldsymbol{p}=\boldsymbol{p}(t)} .
\end{aligned}
$$

From Eq. (III.15) we find for sufficiently large $t$

$$
|\dot{r}(t)| \leqq c|t|^{-(N+1) \alpha}(|r(t)|+1) .
$$

This means that $|\dot{r}(t)|=\mathcal{O}\left(|t|^{-(N+1) \alpha}\right)$ and thus $r(t)$ converges as $t \rightarrow \pm \infty$. Hence the limits $\lim _{t \rightarrow \pm \infty} \Omega_{t}^{-1}$ exist on $\Sigma_{ \pm}$.

If $\left(x_{1}(0), p_{1}(0)\right)$ and $\left(x_{2}(0), p_{2}(0)\right)$ are as stated in the theorem, then Lemma (II.2) gives $\left|\boldsymbol{p}_{1}(t)-\boldsymbol{p}_{2}(t)\right|=\mathcal{O}\left(|t|^{-1-\alpha}\right)$ and thus from Eq. (III.16)

$$
\left|x\left(p_{1}(t), t\right)-x\left(p_{2}(t), t\right)\right|=0\left(|t|^{-\alpha}\right) .
$$

This gives Eq. (III.18), which in combination with Lemma II.2 and Theorem II.1 means that the maps $\Omega_{ \pm}^{-1}$ are $1-1$ and onto. To prove the intertwining property, in Eq. (III.18) let

$$
\left(x_{1}(0), p_{1}(0)\right)=(x(\lambda), p(\lambda)) \quad \text { and } \quad\left(x_{2}(0), p_{2}(0)\right)=(x(0), p(0)) .
$$

Then if $\Omega_{ \pm}^{-1}(x(0), p(0))=(a, p)$

$$
\Omega_{ \pm}^{-1}(x(\lambda), p(\lambda))=\left(a+\lim _{t \rightarrow \pm \infty}(x(t+\lambda)-x(t)), p\right) .
$$

But because of Eq. (II.10), $\boldsymbol{x}(t+\lambda)-\boldsymbol{x}(t) \rightarrow \boldsymbol{p} \lambda$. Hence

$$
\Omega_{ \pm}^{-1} U_{\lambda}(x(0), p(0))=S_{\lambda}^{(0)} \Omega_{ \pm}^{-1}(x(0), p(0))
$$

giving Eq. (III.17). This completes the proof of Theorem III.1.

It remains to show that $\lim _{t \rightarrow \pm \infty} \Omega_{t}=\Omega_{ \pm}$and to prove that the wave operators are canonical transformations. This we do in the next theorem.

Theorem III.2. Assume (III.14) and let $D \Omega_{t}$ be the matrix of derivatives $\frac{\partial \Omega_{t}}{\partial Q_{j}}(Q)_{i}$. The limits $\Omega_{t} \rightarrow \Omega_{ \pm}$and $D \Omega_{t} \rightarrow D \Omega_{ \pm}$are achieved uniformly on compact subsets of $\Sigma$. Since the transformations $\Omega_{t}$ are canonical, the wave 
operators are (contimuously differentiable) canonical transformations on $\Sigma$ :

$$
\left[\Omega_{ \pm}(Q)_{i}, \Omega_{ \pm}(Q)_{j}\right]=\left[Q_{i}, Q_{j}\right] \quad Q \in \Sigma
$$

where $[$,$] is the Poisson bracket.$

The proof will be given for $\Omega_{-}$only.

Proof. It will prove useful in what follows to rewrite Eq. (III.19) as an integral equation. For that purpose, let $\boldsymbol{a}=\lim _{t \rightarrow-\infty} r(t), Q=(\boldsymbol{a}, \boldsymbol{p})$. Then

Let

$$
U_{t} \Omega_{-}(Q)=(\boldsymbol{x}(t), \boldsymbol{p}(t)) .
$$

$$
\beta(Q ; t)=(\boldsymbol{x}(t)-\boldsymbol{x}(\boldsymbol{p}(t), t)-\boldsymbol{a}, \boldsymbol{p}(t)-\boldsymbol{p})=\left(\boldsymbol{\beta}_{1}(Q ; t), \boldsymbol{\beta}_{2}(Q ; t)\right)
$$

and define for each $\alpha=\left(\boldsymbol{\alpha}_{1}, \boldsymbol{\alpha}_{2}\right) \in \Sigma$

$$
\begin{aligned}
\left(G_{1}\right)_{j}(\alpha, s)= & \frac{\partial x_{j}}{\partial \alpha_{2 i}}\left(\alpha_{2}, s\right)\left[F_{i}\left(x\left(\alpha_{2}, s\right)\right)-F_{i}\left(x\left(\alpha_{2}, s\right)+\boldsymbol{\alpha}_{1}\right)\right] \\
& +\frac{\partial}{\partial \alpha_{2 j}}\left(U_{N+1}\left(\boldsymbol{\alpha}_{2}, s\right)-U_{N}\left(\boldsymbol{\alpha}_{2}, s\right)\right) \\
\left(G_{2}\right)_{j}(\alpha, s)= & F_{j}\left(\boldsymbol{x}\left(\boldsymbol{\alpha}_{2}, s\right)+\alpha_{1}\right) .
\end{aligned}
$$

The reason for this long list of definitions is the concise equation satisfied by $\beta(Q ; t)$ :

$$
\beta(Q ; t)=\int_{-\infty}^{t} d s G(Q+\beta(Q ; s), s) .
$$

Suppose $K \subseteq \Sigma$ is compact, and all $(\boldsymbol{a}, \boldsymbol{p}) \in K$ satisfy $|\boldsymbol{a}| \leqq a_{0},|\boldsymbol{p}| \geqq p_{0}>0$. Let $M_{T, \varepsilon}$ be the set of all continuous functions $\gamma:(-\infty, T] \rightarrow \boldsymbol{R}^{6}$ with $\|\gamma\|_{T}=\sup _{s \in(-\infty, T)}|\gamma(s)| \leqq \varepsilon$ where $\varepsilon<p_{0}$.

Then for $T$ sufficiently negative, the operator $\varphi^{Q}$

$$
\varphi^{Q}(\gamma)(t) \equiv \int_{-\infty}^{t} d s G(Q+\gamma(s), s)
$$

maps $M_{T, \varepsilon}$ into $M_{T, \varepsilon}$ and for $\gamma, \gamma^{\prime} \in M_{T, \varepsilon}$

$$
\left\|\varphi^{Q}\left(\gamma^{\prime}\right)-\varphi^{Q}(\gamma)\right\|_{T} \leqq \varrho\left\|\gamma^{\prime}-\gamma\right\|_{T}, \varrho<1
$$

for all $Q \in K$. These statements are easily proved from the estimates

$$
\begin{gathered}
|G(\alpha, s)| \leqq C_{\delta}(1+|s|)^{-\alpha(N+1)} \\
\left|\frac{\partial G}{\partial \alpha}(\alpha, s)\right| \leqq C_{\delta}(1+|s|)^{-\alpha(N+1)}
\end{gathered}
$$


which hold for all $\alpha$ with $\left|\boldsymbol{\alpha}_{1}\right| \leqq \delta^{-1},\left|\boldsymbol{\alpha}_{2}\right| \geqq \delta$ and $\delta$ arbitrary except $\delta>0$. The fact that $\varphi^{Q}$ is a contraction allows us to conclude that $\beta(Q ; t) \in M_{T, \varepsilon}$ because the contraction mapping theorem says that a solution $\gamma(t)$ exists with $\gamma \in M_{T, \varepsilon}$ and Lemma II.2 tells us $\gamma$ and $\beta(Q ; \cdot)$ must be equal.

Consider the function $U_{s} \Omega_{t}(Q)=U_{s-t} U_{t}^{(0)}(Q)=\left(\boldsymbol{x}^{(t)}(s), \boldsymbol{p}^{(t)}(s)\right)$ and the related function

$$
\beta^{t}(Q ; s)=\left(\boldsymbol{x}^{(t)}(s)-\boldsymbol{x}\left(\boldsymbol{p}^{(t)}(s), s\right)-\boldsymbol{a}, \boldsymbol{p}^{(t)}(s)-\boldsymbol{p}\right) .
$$

Note that $\frac{\partial \beta^{t}}{\partial s}(Q ; s)=G\left(Q+\beta^{t}(Q ; s), s\right) ; \beta^{t}(Q ; t)=0$. Thus

$$
\beta^{t}(Q ; s)=\varphi^{Q, t}\left(\beta^{t}(Q ;)\right)(s) \equiv \int_{i}^{s} d \lambda G\left(Q+\beta^{t}(Q ; \lambda), \lambda\right) .
$$

The operator $\varphi^{Q, t}$ also maps $M_{T, \varepsilon} \rightarrow M_{T, \varepsilon}$ and satisfies (III.26) for the same reasons as $\varphi^{Q}$, and in addition the estimate (III.27) implies

$$
\lim _{t \rightarrow-\infty}\left\|\varphi^{Q, t}-\varphi^{Q}\right\|_{T}=0
$$

uniformly in $Q$ for $Q \in K$.

Here $\left\|\varphi^{Q, t}-\varphi^{Q}\right\|_{T}=\sup _{\gamma \in M_{T, \varepsilon}}\left\|\varphi^{Q, t}(\gamma)-\varphi^{Q}(\gamma)\right\|_{T}$. Note that $\beta^{t}(Q ; s) \in M_{T, \varepsilon}$ because the contraction mapping theorem says that a fixed point $\gamma \in M_{T, \varepsilon}$ exists and by the uniqueness of the initial value problem for Newton's equation $\gamma(s) \equiv \beta^{t}(Q ; s)$. Now

$$
\begin{gathered}
\left\|\beta(Q ; \cdot)-\beta^{t}(Q ; \cdot)\right\|_{T}=\left\|\varphi^{Q}(\beta(Q ; \cdot))-\varphi^{Q, t}\left(\beta^{t}(Q ; \cdot)\right)\right\|_{T} \\
\leqq\left\|\varphi^{Q}(\beta(Q ; \cdot))-\varphi^{Q}\left(\beta^{t}(Q ; \cdot)\right)\right\|_{T^{+}}+\left\|\varphi^{Q}\left(\beta^{t}(Q ; \cdot)\right)-\varphi^{Q, t}\left(\beta^{t}(Q ; \cdot)\right)\right\|_{T}
\end{gathered}
$$

and thus

$$
\left\|\beta(Q ; \cdot)-\beta^{t}(Q ; \cdot)\right\|_{T} \leqq(1-\varrho)^{-1}\left\|\varphi^{Q}-\varphi^{Q, t}\right\|_{T} .
$$

Thus in particular $\beta^{t}(Q ; T)-\beta(Q ; T) \rightarrow 0$ uniformly in $Q$ for $Q \in K$ and therefore $U_{T} \Omega_{t}(Q) \rightarrow U_{T} \Omega_{-}(Q)$ as $t \rightarrow-\infty$ uniformly in $Q$. Since $U_{-T}$ is continuous we have

$$
\Omega_{t}(Q) \rightarrow \Omega_{-}(Q) \text { uniformly in } Q \text { for } Q \in K .
$$

The convergence of $D \Omega_{t}(Q)$ is proved similarly: First note that because $\boldsymbol{F}(\boldsymbol{x})$ is continuously differentiable, $\left(\boldsymbol{x}^{(t)}(s), \boldsymbol{p}^{(t)}(s)\right)$ is continuously differentiable in its "initial" value $\left(\boldsymbol{x}^{(t)}(t), \boldsymbol{p}^{(t)}(t)\right)$ [8]. Since the latter is in turn continuously differentiable in $Q, \frac{\partial x_{i}^{(t)}}{\partial Q_{j}}(s)$ and $\frac{\partial p_{i}^{(t)}}{\partial Q_{j}}(s)$ exist and are 
continuous. They also satisfy the linear D.E.

$$
\begin{aligned}
& \frac{d}{d s} \psi_{i j}(s)=A_{i j}(s) \\
& \frac{d}{d s} \Lambda_{i j}(s)=\frac{\partial F_{i}}{\partial x_{k}}\left(x^{(t)}(s)\right) \psi_{k j}(s)
\end{aligned}
$$

(with $\left.\psi_{i j}=\frac{\partial x_{i}^{(t)}}{\partial Q_{j}}, \Lambda_{i j}=\frac{\partial p_{i}^{(t)}}{\partial Q_{j}}\right)$.

The propagation function $L_{s}^{(t)}$ for this D.E.

$$
L_{s}^{(t)}(\psi(0), \Lambda(0))=(\psi(s), \Lambda(s))
$$

has the property that if $\left(\psi^{(t)}(T), \Lambda^{(t)}(T)\right) \underset{t \rightarrow-\infty}{\longrightarrow}(\psi(T), \Lambda(T))$, then

$$
\left(\psi^{(t)}(0), \Lambda^{(t)}(0)\right)=\left(L_{T}^{(t)}\right)^{-1}\left(\psi^{(t)}(T), \Lambda^{(t)}(T)\right) \rightarrow L_{T}^{-1}(\psi(T), \Lambda(T))
$$

uniformly in $Q$ for $Q \in K$ [8]. Here $L_{s}$ results from $L_{s}^{(t)}$ by replacing $\boldsymbol{x}^{(t)}(s)$ by its limit $\boldsymbol{x}(s)$. Note that $L_{s}^{(t)}$ depends on $Q$ through $\boldsymbol{x}^{(t)}(s)$.

Now consider the corresponding quantities $\frac{\partial \beta_{k}^{(t)}}{\partial Q_{i}}(s)$ which satisfy the integral equation

$$
\frac{\partial \beta_{k}^{(t)}}{\partial Q_{j}}(s)=\int_{t}^{s} d \lambda \frac{\partial G_{k}}{\partial Q_{j}}\left(Q+\beta^{(t)}(\lambda), \lambda\right)\left(\delta_{j i}+\frac{\partial \beta_{j}^{(t)}}{\partial Q_{i}}(\lambda)\right)
$$

Using similar methods as in the proof that $\left\|\beta^{t}-\beta\right\|_{T} \rightarrow 0$ uniformly in $Q$ for $Q \in K$ we can show that $\left\|\frac{\partial \beta_{k}^{(t)}}{\partial Q_{i}}-\gamma_{k i}\right\|_{T} \rightarrow 0$ uniformly in $Q$ for $Q \in K$ where $\gamma_{k i}$ is the solution to

$$
\gamma_{k i}(s)=\int_{-\infty}^{s} d \lambda \frac{\partial G_{k}}{\partial Q_{j}}(Q+\beta(\lambda), \lambda)\left(\delta_{j i}+\gamma_{j i}(\lambda)\right)
$$

Thus $\gamma_{k i}(s) \rightarrow \frac{\partial \beta_{k}}{\partial Q_{i}}(s)$ for $s \in(-\infty, T]$. Hence in particular

$$
\frac{\partial x^{(t)}}{\partial Q_{i}}(T) \rightarrow \frac{\partial x(T)}{\partial Q_{i}}, \frac{\partial p^{(t)}}{\partial Q_{i}}(T) \rightarrow \frac{\partial p(T)}{\partial Q_{i}}
$$

The properties of $L_{s}^{(t)}$ mentioned after Eq. (III.14) then show the uniform convergence of $\frac{\partial \Omega_{t}}{\partial Q_{i}}(Q)$. The Poisson brackets $\left[\Omega_{t}(Q)_{i}, \Omega_{t}(Q)_{j}\right]=\left[Q_{i}, Q_{j}\right]$ thus also converge to $\left[\Omega_{-}(Q)_{i}, \Omega_{-}(Q)_{j}\right]$. This proves Theorem III.2. 
Theorem III.1 and III.2 give the wave operators many of the familiar properties they enjoy in Q.M. The isometric nature of the wave operators in Q.M. has its analog in their being canonical transformations in classical mechanics. The fact that they are not in general unitary in Q.M. has its analog in the fact that the range of $\Omega_{ \pm}$is missing just those initial conditions corresponding to bound orbits (up to a set of Lebesgue measure zero) ${ }^{1}$.

It is interesting to note that while $\Omega_{ \pm}=\lim _{t \rightarrow \pm \infty} U_{-t} U_{t}^{(0)}$, the equation $U_{t} \Omega_{ \pm}-U_{t}^{(0)} \rightarrow 0$ is characteristically false in the long range case. (This is the exact opposite of the situation with $W_{ \pm}$as Eq. (II.35) partially verifies.) In fact the above limit is characteristically $\infty$. This suggests the question as to how these properties manifest themselves in Q.M. We feel that many of the convergence statements in this paper will be true in the sense of convergence of the appropriately transformed $\boldsymbol{x}$ or $\boldsymbol{p}$ in Q.M. Thus for example we conjecture that for the quantum mechanical operators $\Omega_{t}$ constructed by Buslaev and Matveev [1]

$$
\begin{aligned}
& \Omega_{t}^{-1}(x) \equiv \Omega_{t} x \Omega_{t}^{* s} \Omega_{ \pm} x \Omega_{ \pm}^{*} \\
& \Omega_{t}^{-1}(p) \equiv \Omega_{t} p \Omega_{t}^{* s} \rightarrow \Omega_{ \pm} p \Omega_{ \pm}^{*}
\end{aligned}
$$

on a dense set of states in the range of $\Omega_{ \pm}$. This is the analog of Theorem III.1. Indeed $\Omega_{t} \boldsymbol{x} \Omega_{t}^{*}=e^{i \boldsymbol{H} t}\left(\boldsymbol{x}-\boldsymbol{x}_{N}(\boldsymbol{p}, t)\right) e^{-i \boldsymbol{H} t}=\boldsymbol{x}(t)-\boldsymbol{x}_{N}(\boldsymbol{p}(t), t)$ where $\boldsymbol{x}(t)$ and $\boldsymbol{p}(t)$ are Heisenberg operators and $\boldsymbol{x}_{N}(\boldsymbol{p}, t)$ is the function introduced in Eq. (III.13). Thus formally $\Omega_{t} x \Omega_{t}^{*}$ is the same as the function $r(t)$ introduced in the proof of Theorem III.1.

On the other hand the fact that $U_{t} \Omega_{ \pm}-U_{t}^{(0)} \nrightarrow 0$ should manifest itself in the following way: If $\psi=\Omega_{-} f$, we know that [1] $e^{-i H t} \psi{ }^{s} \rightarrow U_{t}^{(0)} f$, but we conjecture that characteristically

$$
(\psi, \boldsymbol{x}(t) \psi)-\left(U_{t}^{(0)} f, \boldsymbol{x} U_{t}^{(0)} f\right) \nrightarrow 0
$$

(while of course the above always has limit zero for bounded functions of $\boldsymbol{x}$ ).

Before going on to examine what kind of spatial cluster properties a long range interaction has, we must define the $S$-operator. The definition

$$
S=\Omega_{+}^{-1} \Omega_{-}
$$

is conventional. It has the consequence that the domain and range of $S$ are respectively $\Omega_{-}^{-1}\left(\Sigma_{+} \cap \Sigma_{-}\right)$and $\Omega_{+}^{-1}\left(\Sigma_{+} \cap \Sigma_{-}\right)$. As a result of the next theorem, the latter two sets differ from $\boldsymbol{R}^{6}$ by sets of measure zero.

${ }^{1}$ For a justification of the measure theoretic part of this statement, see Theorem III.3 below. 
Theorem III.3 (Asymptotic Completeness). Assume (II.2) through (II.4). Then $\Sigma_{+}=\Sigma_{-}$up to a set of Lebesgue measure zero (i.e. $\Sigma_{+} \cup \Sigma_{-}$ $-\Sigma_{+} \cap \Sigma_{-}$has measure zero).

The proof of this result can be found in [7] or [9].

\section{Cluster Properties of the $S$-operator}

Let $T(a): R^{6} \rightarrow R^{6}$ be a translation of the $x$ coordinate of a point in phase space:

$$
T(\boldsymbol{a})(\boldsymbol{x}, \boldsymbol{p})=(\boldsymbol{x}+\boldsymbol{a}, \boldsymbol{p}) .
$$

We want to consider the behavior of $T(-\boldsymbol{a}) S T(\boldsymbol{a})$ for large $|\boldsymbol{a}|$. This behavior is very different for short range and for long range forces. We will consider the short range case first. We define a short range force by the following conditions, which are essentially those of Ref. [5]

$$
\begin{gathered}
\boldsymbol{F}(\boldsymbol{x})=-(\boldsymbol{\nabla} V)(\boldsymbol{x}), \quad \lim _{\boldsymbol{x} \rightarrow \infty} V(\boldsymbol{x})=0, \\
|\boldsymbol{F}(\boldsymbol{x})| \leqq k(1+|\boldsymbol{x}|)^{-(2+\varepsilon)} ; \quad \varepsilon>0, \\
\boldsymbol{F} \in C^{1}\left(R^{3}\right) \quad \text { and }\left|\frac{\partial F_{i}}{\partial x_{j}}\right| \leqq k(1+|\boldsymbol{x}|)^{-(2+\varepsilon)} .
\end{gathered}
$$

Then $\Omega_{ \pm}=\lim _{t \rightarrow+\infty} U_{-t} S_{t}^{(0)}$ exists and is a (canonical) transformation of $\Sigma$ onto $\Sigma_{ \pm}$(see Ref. [5]). With the $S$-operator defined as in (III.39) we then have the following theorem.

Theorem IV.1. Suppose V(x) satisfies (IV.2) through (IV.4). Let $K \subseteq \Sigma$ be compact and suppose $(\boldsymbol{x}, \boldsymbol{p}) \in K \Rightarrow p \in \Gamma, \Gamma$ a closed cone with apex at $p=0$ and solid angle $<4 \pi$. For $(x, p) \in K$, let

$$
(\boldsymbol{x}(\boldsymbol{a}), \boldsymbol{p}(\boldsymbol{a})) \equiv T(-\boldsymbol{a}) S T(\boldsymbol{a})(\boldsymbol{x}, \boldsymbol{p}) .
$$

Then as $a \rightarrow \infty$ outside any open cone $\Gamma_{\delta} \supseteqq \Gamma$

$$
\lim _{|\boldsymbol{a}| \rightarrow \infty}(T(-\boldsymbol{a}) S T(\boldsymbol{a})-I)=0
$$

uniformly on $K$. In fact

$$
\begin{aligned}
& |\boldsymbol{x}(\boldsymbol{a})-\boldsymbol{x}|=\mathcal{O}\left(|\boldsymbol{a}|^{-\varepsilon}\right) \\
& |\boldsymbol{p}(\boldsymbol{a})-\boldsymbol{p}|=\mathcal{O}\left(|\boldsymbol{a}|^{-1-\varepsilon}\right)
\end{aligned}
$$

uniformly in $(\boldsymbol{x}, \boldsymbol{p})$ for $(\boldsymbol{x}, \boldsymbol{p}) \in K$.

We defer the proof of this theorem (and Theorem IV.2 to follow) until the end of this section. 
Because of Eq. (IV.7), Theorem IV.1 says that the rate of approach of $T(-a) S T(a)-I$ to zero is a measure of just how short range the forces are. For long range forces Theorem IV.1 is false, but the corresponding cluster property still gives a measure of the rate of decrease of $\boldsymbol{F}(\boldsymbol{x})$.

Theorem IV.2. Suppose $V(x)$ satisfies (III.14). Then with $K, \Gamma$, and $\Gamma_{\delta}$ as in Theorem IV.1 and

$$
(x(a), p(a))=T(-a) S T(a)(x, p),
$$

then as $a \rightarrow \infty$ outside $\Gamma_{\delta}$

$$
\begin{aligned}
& |p(a)-p|=\mathcal{O}\left(|a|^{-\alpha}\right) \\
& |x(a)-x|=\mathcal{O}\left(|a|^{1-\alpha}\right)
\end{aligned}
$$

uniformly in $(\boldsymbol{x}, \boldsymbol{p})$ for $(\boldsymbol{x}, \boldsymbol{p}) \in K$.

In order to get a feeling for the behavior of $\boldsymbol{x}(\boldsymbol{a})$ as defined above, we specialize to the class of potentials for which $\alpha>\frac{1}{2}$.

Theorem IV.3. Suppose V(x) satisfies (III.14) with $N=[1 / \alpha]=1$. Then with $K, \Gamma$, and $\Gamma_{\delta}$ as above,

$$
\boldsymbol{x}(\boldsymbol{a})=\boldsymbol{x}+\boldsymbol{\nabla}_{\boldsymbol{p}} \int_{-\infty}^{\infty} d t(V(\boldsymbol{p} t+\boldsymbol{a})-V(\boldsymbol{p} t))+\mathcal{O}\left(|\boldsymbol{a}|^{1-2 \alpha}\right)
$$

as $\boldsymbol{a} \rightarrow \infty$ outside $\Gamma_{\delta}$, uniformly in $(\boldsymbol{x}, \boldsymbol{p})$ for $(\boldsymbol{x}, \boldsymbol{p}) \in K$.

We remark that $\boldsymbol{x}(\boldsymbol{a})-\boldsymbol{x}$ characteristically diverges as $\boldsymbol{a} \rightarrow \infty$. Thus in Q.M. we expect the following behavior for the amplitude

$$
(T(a) f, S T(a) g) \text {. }
$$

As $|\boldsymbol{a}|$ gets large, $g(\boldsymbol{a}) \equiv T(-\boldsymbol{a}) S T(\boldsymbol{a}) g$ becomes a state with the same momentum space probability distribution as $g$ (Theorem IV.2), but in $\boldsymbol{x}$ space $g(\boldsymbol{a})$ has moved very far from $g$ (and $f$ ). Thus the overlap of $g(\boldsymbol{a})$ with $f$ in $x$-space is very small. Hence we expect that

$$
\lim _{|a| \rightarrow \infty}(T(a) f, \dot{S} T(a) g)=0 .
$$

Indeed this has been verified for the non-relativistic Coulomb $S$-operator by Ross [12].

In an Appendix we discuss the spatial cluster property of the classical $S$-operator for the Coulomb scattering problem and compare our results with those of Ross.

Proof of Theorem IV.1. Suppose $Q \in \Sigma$. We consider the quantity $\beta(Q ; t)$ (compare Eq. (III.22)) given by

$$
\beta(Q ; t)=\left(\Omega_{t}^{-1} \Omega_{-}-I\right)(Q) .
$$


Note that since $\Omega_{t}=U_{t}^{-1} S_{t}^{(0)}$ we have

$$
\beta(Q ; t)=\left(\boldsymbol{\beta}_{1}(Q ; t), \boldsymbol{\beta}_{2}(Q ; t)\right)=\left(\boldsymbol{x}(t)-t \boldsymbol{p}(t)-\boldsymbol{Q}_{1}, \boldsymbol{p}(t)-\boldsymbol{Q}_{2}\right)(\mathrm{IV}
$$

where here

$$
(\boldsymbol{x}(t), \boldsymbol{p}(t))=U_{t} \Omega_{-}(Q), \quad Q=\left(Q_{1}, Q_{2}\right) .
$$

Since $\Omega_{ \pm}^{-1}=\lim _{t \rightarrow \pm \infty} \Omega_{t}^{-1}$ (the analog of Theorem III.1)

$$
\lim _{t \rightarrow-\infty} \beta(Q ; t)=0 \text {. }
$$

Note also that for the same reason, if $Q$ is in the domain of $S$

$$
\beta(Q) \equiv \lim _{t \rightarrow+\infty} \beta(Q ; t)=(S-I)(Q)
$$

Using the fact that $S_{\lambda}^{(0)}$ commutes with $S$ one easily derives the equality

$$
\beta\left(Q_{1}+\lambda Q_{2}, Q_{2}\right)=\beta(Q)+\left(\lambda \beta_{2}(Q), 0\right)
$$

valid also in the sense that $\left(Q_{1}+\lambda Q_{2}, Q_{2}\right)$ is in the domain of $S$ if and only if $Q$ is.

If $Q=(\boldsymbol{a}, \boldsymbol{p}) \in \Sigma$ with $\boldsymbol{a} \cdot \boldsymbol{p}=0$, we will derive the following estimates which hold for $|\boldsymbol{p}| \geqq p_{0}>0$ and all $t$ if $|\boldsymbol{p}|^{2}|\boldsymbol{a}|^{1+\varepsilon}$ is large enough:

$$
\begin{aligned}
& \left|\boldsymbol{\beta}_{1}(Q ; t)\right| \leqq c|p|^{-2}|\boldsymbol{a}|^{-\varepsilon} \\
& \left|\boldsymbol{\beta}_{2}(Q ; t)\right| \leqq c|\boldsymbol{p}|^{-1}|\boldsymbol{a}|^{-1-\varepsilon} .
\end{aligned}
$$

To see that this is enough to prove the theorem first note that

$$
|\boldsymbol{x}(t)| \geqq|\boldsymbol{p} t+\boldsymbol{a}|-\left|t \boldsymbol{\beta}_{2}(Q ; t)\right|-\left|\boldsymbol{\beta}_{1}(Q ; t)\right|
$$

and thus if $p^{2} a^{1+\varepsilon}>c$ the estimates (IV.17) imply

$\varlimsup_{t \rightarrow+\infty}|\boldsymbol{x}(t)| \geqq \varlimsup_{t \rightarrow+\infty}\left\{|\boldsymbol{p} t|\left(1-c\left(p^{2} a^{1+\varepsilon}\right)^{-1}\right)-\left|\boldsymbol{\beta}_{2}(Q ; t)\right|\right\}=\infty$.

Hence if $p^{2} a^{1+\varepsilon}$ is large enough $Q$ is in the domain of $S$ (Lemma II.1). Now consider $(\boldsymbol{x}, \boldsymbol{p}) \in K$ and the expression

$$
(T(-a) S T(a)-I)(x, p)=\beta(x+a, p)
$$

where the last equality follows from the definitions of $\beta(Q)$ and $T(a)$. We split up $\boldsymbol{x}$ and $\boldsymbol{a}$ into

$$
\begin{aligned}
& \boldsymbol{x}=\boldsymbol{x}_{\perp}+\lambda_{x} \boldsymbol{p} \\
& \boldsymbol{a}=\boldsymbol{a}_{\perp}+\lambda_{a} \boldsymbol{p}
\end{aligned}
$$

where $\boldsymbol{x}_{\perp}$ and $\boldsymbol{a}_{\perp}$ are perpendicular to $\boldsymbol{p}$. Note that because $K \cong \Sigma$ and compact, $x_{\perp}$ and $\lambda_{x}$ vary in compact sets. Note also that if $a \in \Gamma_{\delta},\left|\boldsymbol{a}_{\perp}\right| \geqq \gamma\left|\lambda_{a}\right|$ 
with $\gamma$ independent of $p$. Thus since

$$
\beta(\boldsymbol{x}+\boldsymbol{a}, \boldsymbol{p})=\beta\left(\boldsymbol{x}_{1}+\boldsymbol{a}_{\perp}, \boldsymbol{p}\right)+\left(\lambda_{x}+\lambda_{a}\right)\left(\boldsymbol{\beta}_{2}\left(\boldsymbol{x}_{\perp}+\boldsymbol{a}_{\perp}, \boldsymbol{p}\right), \mathbf{0}\right),
$$

if $|\boldsymbol{a}|$ is large enough $(\boldsymbol{x}+\boldsymbol{a}, \boldsymbol{p})$ is in the domain of $S$ for all $(\boldsymbol{x}, \boldsymbol{p}) \in K$, and the estimates (IV.17) give

$$
\begin{aligned}
& \left|\boldsymbol{\beta}_{1}(\boldsymbol{x}+\boldsymbol{a}, \boldsymbol{p})\right| \leqq c|\boldsymbol{p}|^{-2}\left|\boldsymbol{x}_{\perp}+\boldsymbol{a}_{\perp}\right|^{-\varepsilon}+\left|\lambda_{\boldsymbol{x}}+\lambda_{a}\right| c|\boldsymbol{p}|^{-1}\left|\boldsymbol{x}_{\perp}+\boldsymbol{a}_{\perp}\right|^{-1-\varepsilon} \leqq c_{1}|\boldsymbol{a}|^{-\varepsilon} \\
& \left|\boldsymbol{\beta}_{2}(\boldsymbol{x}+\boldsymbol{a}, \boldsymbol{p})\right| \leqq c|\boldsymbol{p}|^{-1}\left|\boldsymbol{x}_{\perp}+\boldsymbol{a}_{\perp}\right|^{-1-\varepsilon} \leqq c_{2}|\boldsymbol{a}|^{-1-\varepsilon} .
\end{aligned}
$$

Thus the theorem is proved once we have derived the estimates (IV.17) under the stated conditions.

Consider the quantity

$$
C(t)=p^{2} a^{\varepsilon}\left|\boldsymbol{\beta}_{1}(t)\right|+p a^{1+\varepsilon}\left|\boldsymbol{\beta}_{2}(t)\right|
$$

where $\beta(t) \equiv \beta(Q ; t), Q=(\boldsymbol{a}, \boldsymbol{p}), \boldsymbol{a} \cdot \boldsymbol{p}=0$. We know that $C(t) \rightarrow 0$ as $t \rightarrow-\infty$. We will show that there exists $k_{0}$ such that for all $(\boldsymbol{a}, \boldsymbol{p})$ satisfying $p^{2} a^{1+\varepsilon}>$ const.

$$
C(t)<k_{0} \text { all } t \text {. }
$$

The proof proceeds in the following way. Suppose $k_{0}>0, \eta>1$ and $p^{2} a^{1+\varepsilon}>\sqrt{2} k_{0} \eta$. Then if $C(t)>k_{0}$ for some $t$, the continuity of $C(t)$ implies $C(t)<k_{0}$ for $t \in(-\infty, T)$ and $C(T)=k_{0}$ for some $T$. For certain choices of $k_{0}$ and $\eta$ we will show that the conditions $C(t)<k_{0}$ for $t \in(-\infty, T)$ and $p^{2} a^{1+\varepsilon}>\sqrt{2} k_{0} \eta$ imply $C(T)<k_{0}$. This contradiction will prove (IV.23). Thus we first note that

$$
\begin{aligned}
& \boldsymbol{\beta}_{1}(t)=-\int_{-\infty}^{t} d s s \boldsymbol{F}(\boldsymbol{x}(s)) \\
& \boldsymbol{\beta}_{2}(t)=\int_{-\infty}^{t} d s \boldsymbol{F}(\boldsymbol{x}(s))
\end{aligned}
$$

and that $C(t)<k_{0}$ for $t \in(-\infty, T)$ implies (see (IV.18))

$$
|\boldsymbol{x}(s)|>(|\boldsymbol{a}|+|\boldsymbol{p} s|) \xi, \quad s \in(-\infty, T)
$$

where $\xi \equiv(1 / \sqrt{2})\left(1-\sqrt{2} k_{0} / p^{2} a^{1+\varepsilon}\right)>0$.

Using (IV.24) and (IV.3) we can estimate $C(T)$ with the result

$$
C(T)<2 k(1+2 \varepsilon)\left(\varepsilon(1+\varepsilon) \xi^{2+\varepsilon}\right)^{-1}
$$

where $k$ is the constant appearing in (IV.3). Since as $p^{2} a^{1+\varepsilon} \rightarrow \infty \xi \rightarrow 1 / \sqrt{2}$, for any $k_{0}>2 k(1+2 \varepsilon)(\varepsilon(1+\varepsilon))^{-1} 2^{1+\varepsilon / 2}$ we can find $\eta$ such that if $p^{2} a^{1+\varepsilon}>\sqrt{2} \eta k_{0}$, (IV.26) implies $C(T)<k_{0}$. This completes the proof of Theorem IV.1. 
Proof of Theorem $I V .2$. Because the proof is very similar to that of Theorem IV.1 we only sketch it. With $Q=(a, p) \in \Sigma$ and $a \cdot p=0$, define $\beta(t)$ as in Eq. (IV.11). Then $\beta$ satisfies (III.24). With

$$
C(t)=a^{\frac{1}{2} \alpha-1}\left|\boldsymbol{\beta}_{1}(t)\right|+\left|\boldsymbol{\beta}_{2}(t)\right|
$$

an argument similar to the preceding one gives $C(t)<\varepsilon$ for any $\varepsilon>0$ and all $t$ if $a>a(\varepsilon)$. Using this information and Eq. (III.24) one can again estimate $\beta(t)$ with the result given in the theorem.

We do not give the proof of Theorem IV.3 for it involves only straightforward but tedious estimates.

\section{Concluding Remarks}

We have shown in Section II that a very reasonable classical scattering theory exists for slowly decreasing potentials with only mild restrictions on the forces. However canonical wave operators (and thus a canonical $S$-operator) were constructed (in Section III) only after a considerable strengthening of the assumptions on the forces. It remains to be seen whether canonical $S$-operators in classical mechanics (and thus presumably unitary $S$-operators in quantum potential scattering) can be constructed without these restrictive assumptions on the forces. We remark that for central potentials the operators $W_{ \pm}$of Section II can be shown to be canonical transformations (under further mild restrictions on the potential), but we have not tried the analogous construction in Q.M.

Acknowledgements. I would like to thank G. W. Ford, B. Simon, and V. Wong for helpful discussions. It is a pleasure to thank D. N. Williams for his critical reading of the manuscript and constructive suggestions.

\section{Appendix: Cluster Property for the Coulomb Potential}

Because the Coulomb potential, $V(\boldsymbol{x})=\lambda|\boldsymbol{x}|^{-1}$, has a singularity at $\boldsymbol{x}=0$, the results of this paper are not directly applicable. The main difficulty is the fact that the quasi-free time evolution, $U_{t}^{(0)}$, as defined in (III.3), (III.4), and (III.13) (with $N \equiv[1 / \alpha]=1$ ) does not exist. However, this is easily remedied by taking for example

$$
H_{0}+V(\boldsymbol{p} t) \theta(|\eta t|-1)
$$

as the Hamiltonian associated with $U_{t}^{(0)}$ instead of $H_{0}+V(p t)$. The $\theta$-function cutoff removes the singularity at $t=0$ and thus $U_{t}^{(0)}$ exists. Although the kind of cutoff used is essentially arbitrary, Eq. (A.1) with 
$\eta=4 H_{0}=2 p^{2}$ is the one which in Q.M. gives the standard result for the Coulomb $S$-matrix [2] and is therefore the one we will use. With this prescription, for $2 p^{2}|t|>1$

where

$$
U_{t}^{(0)}(\boldsymbol{a}, \boldsymbol{p})=(\boldsymbol{a}+\boldsymbol{x}(\boldsymbol{p}, t), \boldsymbol{p})
$$

$$
\begin{aligned}
\boldsymbol{x}(\boldsymbol{p}, t) & =\boldsymbol{p} t+\nabla_{\boldsymbol{p}} \int_{0}^{t} d s \theta\left(4 H_{0}|s|-1\right) V(\mathbf{p} s) \\
& =\boldsymbol{p} t-\varepsilon(t) \lambda \boldsymbol{p}|\boldsymbol{p}|^{-3} \log \left(4 H_{0}|t| / e^{2}\right) .
\end{aligned}
$$

Here $\varepsilon(t)= \pm 1$ if $\pm t>0$ and $e$ is the base of natural logarithms.

With this choice of $U_{t}^{(0)}$, and $\Omega_{t}=U_{t}^{-1} U_{t}^{(0)}$, Theorems III.1 and III.2 remain true if $\Sigma_{ \pm}$and $\Sigma$ are redefined to exclude points with zero angular momentum.

The integral (IV.9) of Theorem IV.3 must be replaced by

$$
\int_{-\infty}^{\infty} d t\left\{V(\boldsymbol{p} t+\boldsymbol{a})-V(\boldsymbol{p} t) \theta\left(4 H_{0}|t|-1\right)\right\}
$$

and thus the cluster property for the Coulomb potential takes the following form (with $\left.\Sigma=\left\{(a, p) \in R^{6}: a \times p \neq 0\right\}\right)$ : .

Proposition. Suppose $V(x)=\lambda /|x|$ and $U_{t}^{(0)}$ is defined as in (A.2) and (A.3). Let $K \subseteq \Sigma$ be compact and suppose $(\boldsymbol{x}, \boldsymbol{p}) \in K \Rightarrow p \in \Gamma, \Gamma$ a closed cone with apex at $p=0$ and solid angle $<4 \pi$. For $(x, p) \in K$, let

$$
(x(a), p(a))=T(-a) S T(a)(x, p) .
$$

Then as $\boldsymbol{a} \rightarrow \infty$ outside any open cone $\Gamma_{\delta} \supseteqq \Gamma$

$$
\begin{gathered}
|\boldsymbol{p}(\boldsymbol{a})-\boldsymbol{p}|=\mathcal{O}\left(|\boldsymbol{a}|^{-1}\right) \\
\boldsymbol{x}(\boldsymbol{a})=\boldsymbol{x}+\nabla_{\boldsymbol{p}}\left\{-\lambda|\boldsymbol{p}|^{-1} \log |\boldsymbol{a} \times \boldsymbol{p}|^{2}\right\}+\mathcal{O}\left(|\boldsymbol{a}|^{-1} \log |\boldsymbol{a}|\right)
\end{gathered}
$$

uniformly in $(x, p)$ for $(x, p) \in K$.

To compare this result with that of Ross [12], we rewrite his result (after correcting a sign error) in the form

$$
T(-\boldsymbol{a}) S T(\boldsymbol{a}) \stackrel{s}{\rightarrow} e^{i \lambda|\boldsymbol{p}|^{-1} \log |\boldsymbol{a} \times \boldsymbol{p}|^{2}} \equiv W(\boldsymbol{a}, \boldsymbol{p}) .
$$

Eq. (A.7) is true in the sense that the difference between the right and left sides converges strongly to zero as $|\boldsymbol{a}| \rightarrow \infty$. Thus in the limit $\boldsymbol{a} \rightarrow \infty$, $T(-\boldsymbol{a}) S T(\boldsymbol{a}) f \cong W(\boldsymbol{a}, \boldsymbol{p}) f$ has the same momentum spectrum as $f$ but in $\boldsymbol{x}$-space has been slifted. Specifically

$$
\begin{gathered}
(W(\boldsymbol{a}, \boldsymbol{p}) f, \boldsymbol{x} W(\boldsymbol{a}, \boldsymbol{p}) f) \\
=\left(f,\left\{\boldsymbol{x}+\nabla_{\boldsymbol{p}}\left[-\lambda|\boldsymbol{p}|^{-1} \log |\boldsymbol{a} \times \boldsymbol{p}|^{2}\right]\right\} f\right)
\end{gathered}
$$

in agreement with (A.6). 
We remark that the asymptotic difference in the "in" and "out" impact parameters is very different from the formula (A.6). If $s_{1}$ and $s_{2}$ are respectively the in and out impact parameters we have

$$
\begin{aligned}
& s_{1}=x+a-(x+a) \cdot \hat{p} \hat{p} \\
& s_{2}=x(a)+a-(x(a)+a) \cdot \hat{p}(a) \hat{p}(a)
\end{aligned}
$$

and from any textbook on classical mechanics it follows that

$$
\lim _{|\boldsymbol{\alpha} \times \hat{\boldsymbol{p}}| \rightarrow \infty}\left(s_{2}-s_{1}\right)=-2 \lambda|\boldsymbol{p}|^{-3} \boldsymbol{p} .
$$

Note that we have used the remark made after Theorem III.1 to write down (A.9).

\title{
References
}

1. Buslaev, V.S., Matveev, V.B.: Theor. Math. Phys. 1, 367 (1970) (English translation)

2. Dollard, J. D.: J. Math. Phys. 5, 729 (1964)

3. Amrein, W.O., Martin, Ph.A., Misra, B.: Helv. Phys. Acta 43, 313 (1970)

4. Alsholm, P., Kato, T.: Lecture given by second named author at the Am. Math. Soc. Summer Institute on Partial Differential Equations, Berkeley 1971, (to be published)

5. Simon, B.: Commun. math. Phys. 23, 37 (1971)

6. Cook,J.M.: In 1965 Cargese Lectures in Theoretical Physics, F. Lurcat, ed., New York: Gordon and Breach, 1967

7. Hunziker, W.: Commun. math. Phys. 8, 282 (1968)

8. Birkhoff, G., Rota, G.: Ordinary Differential Equations. Boston: Ginn and Co. 1962

9. Siegel,C.L.: Vorlesungen über Himmelsmechanik. Berlin-Göttingen-Heidelberg: Springer 1956

10. Hunziker, W.: J. Math. Phys. 6, 6 (1965)

11. Hepp, K.: In 1965 Brandeis University Summer Institute in Theoretical Physics, Axiomatic Field Theory, ed. M. Chretian and S. Deser. New York: Gordon and Breach 1966

12. Ross, W.: Ph. D. Thesis, University of Colorado, Boulder (1968)

\author{
I. W. Herbst \\ Department of Physics \\ Princeton University \\ Princeton, N.J. 08540, USA
}

